\title{
Exploring Factors Relating to Positive Outcomes for Youth with Bipolar Spectrum Disorder: A Multi-Informant, Mixed Methods Study
}

\author{
Brae Anne McArthur ${ }^{1}$ - Kathlyn M. Cherry ${ }^{2}$. \\ Margaret N. Lumley ${ }^{2}$
}

\begin{abstract}
Bipolar Spectrum Disorder (BSD) is a severe psychiatric disorder, and relatively little is known about positive outcomes for youth with this diagnosis. Employing a multi-informant, mixed-methods approach, this study sought to a) gain a deeper understanding of positive factors identified by youth with a diagnosis of BSD and their caregivers; and b) understand how the parenting context relates to positive functioning among youth with a diagnosis of BSD. A clinical sample of 18 families with a youth diagnosed with BSD participated, completing measures of parenting style, resiliency, positive schemas, mania, depression, and a telephone interview with a qualitative component. Qualitative analyses revealed most caregivers described their youth with BSD as having strong interpersonal relationship qualities and positive internal characteristics. Youth responses cohered largely with caregiver responses. Parental acceptance related to all measures of positive functioning, and results further suggested positive schemas may be one mechanism linking parental acceptance to resiliency for youth with BSD. Despite the severity of a BSD diagnosis, caregivers and youth reported on many positive aspects of their experiences. These results also inform a more complex view of the relation between parenting and resiliency for youth with BSD. With replication, the findings from this preliminary sample may inform the design of future measures of positive functioning for youth with BSD and inform the development of treatment and intervention programs for families coping with BSD.
\end{abstract}

Keywords Pediatric Bipolar Disorder Positive psychology · Adolescent mental health Parenting $\cdot$ positive schemas $\cdot$ Mixed methods

Brae Anne McArthur bmcarthur@temple.edu

1 Psychology Department, Temple University, Weiss Building, Philadelphia, PA 19122, USA

2 Psychology Department, University of Guelph, Guelph, ON, Canada 


\section{Introduction}

Compared to adults, youth diagnosed with Bipolar Spectrum Disorder (BSD) evidence more chronic symptoms, a faster rate of change between manic and depressive states (i.e., rapid cycling), and greater dysphoria (i.e., intense feelings of depression) and irritability (Biederman et al. 2005; Geller and Luby 1997; Youngstrom et al. 2008). Given the deleterious trajectory for youth with BSD (see Alloy et al. 2015; Stange et al. 2015), it is important to identify factors associated with positive developmental outcomes for this population. The goal of this study was to explore factors relating to positive outcomes for youth with BSD, and to examine the role parenting may play in this process.

\section{Positive Clinical Psychology and Youth Psychopathology}

Wood and Tarrier (2010) have stressed the importance of not only acknowledging the difficulties and negative aspects of functioning associated with psychopathology (e.g., sadness, anxiety, anger, stress, loneliness, low self-esteem, etc.), but also the positive aspects of functioning (e.g., gratitude, flexibility, positive emotions, well-being, life satisfaction, resiliency, etc.) that can aid in predicting disorders, buffering against the negative impact of disorders, and ultimately preventing the development of disorders. This unified framework and commensurate research perspective integrates both positive and negative aspects of experience to better and more holistically understand and treat psychopathology (Wood and Tarrier 2010).

To date, much positive psychology research has focused on non-clinical samples (Larson 2000; Taylor and Brown 1994) with less focus on clinical samples (Adler et al. 1998; Folkman 1997), and even less specifically on those with BSD (Duckworth et al. 2005; Galvez et al. 2011). Further, past research on positive functioning among youth with psychopathology has focused mainly on predetermined concepts of well-being (e.g., life satisfaction, resiliency, positive schemas) and such practices may miss important aspects of positive functioning from the perspective of the youth and/or their caregiver. Taken together, this study's multi-informant, mixed-methods approach to examining positive characteristics and functioning for youth with BSD is therefore quite novel.

\section{Perspectives on Positive Functioning and BSD}

There have been recent calls to include qualitative approaches alongside quantitative methods to gain a more comprehensive understanding of phenomena under study (Armstrong et al. 2005; Braun and Clarke 2006; Krahn et al. 1995). The current study therefore included a qualitative component to gain a deeper understanding of youth and caregiver perspectives on positive functioning for youth with BSD, allowing for responses that were not constrained by prescribed quantitative research measures.

Previous qualitative research has primarily focused on understanding the negative aspects associated with a diagnosis of BSD, such as the impact of manic and depressive states on general functioning and quality of life (Crowe et al. 2012; Fletch et al. 2013; Michalak et al. 2006). A review of the literature yielded only one qualitative study 
examining positive factors associated with having a diagnosis of BSD (Lobban et al. 2012). In this study, ten adults with a diagnosis of BSD (aged 24 to 57) were asked about their understanding of BSD, what difference a diagnosis of BSD has made in their lives, and aspects of their diagnosis they would miss if absent. The results suggested adults with BSD identified themselves as having amplified internal states, enhanced abilities, and more human connectedness than those without this diagnosis. They also identified feeling lucky to have BSD and often saw it as a "special gift" (Lobban et al. 2012). Although this study provides some qualitative information about positive aspects of living with BSD, it focuses solely on an adult population, and youth perspectives are required to extend and enrich such findings.

\section{Resiliency and Positive Schemas as Markers of Positive Functioning}

Two theoretically-established markers of positive functioning were also explored in the current study: resiliency and positive schemas. Overall, the study of resiliency is related to understanding the "process of, capacity for, or pathways and patterns of positive adaptation during or following significant threats to disturbance" (Masten 2011, p. 494). Surprisingly, despite the complex risks associated with $\mathrm{BSD}$, and the potential for knowledge of factors underlying resiliency to be relevant for intervention, this topic has been neglected to date.

Schemas are defined here as mental frameworks that are used to interpret, categorize, and evaluate one's experiences (James et al. 2007; Schmidt et al. 1999). Extant schema and emotional functioning research has mainly focused on negative schemas or maladaptive core beliefs such as "I am defective" or "others cannot be trusted" (e.g., Hawke et al. 2011; Nilsson et al. 2010; Young et al. 2003) and cognitive models generally position schemas as mediating the relation between a negative event or circumstance and depression outcomes (Cole and Turner 1993).

Although the majority of research has focused on linking negative schemas to psychopathology, some research underscores the need for positive schemas (e.g., core beliefs such as, "I am worthy", "Things generally work out") to maintain everyday happiness in normative samples (Cacioppo et al. 1999; Fleming and Darley 1986; Greenwald 1980; Langer and Roth 1975). There is also evidence that positive schemas may be inversely related to psychopathology (Keyfitz et al. 2013; Prieto et al. 1992: MacLeod and Moore 2000; Shirk et al. 1998). When considering depression specifically, depression is often qualified by both the presence of negative schemas and the absence of positive schemas (MacLeod and Byrne 1996; MacLeod and Moore 2000; MacLeod and Salaminiou 2001). Some even suggest a lack of positive schemas may be more important than the presence of negative schemas in the development of youth depression (McClain and Abramson 1995; Whitman and Leitenberg 1990).

To date, positive schemas have not been explored among youth with BSD and this may represent an important gap in the literature. Despite evidence from the adult literature suggesting that adults with BSD show signs of well-being (e.g., creativity, reward motivation, achievement motivation, goal setting, empathy, etc.; Galvez et al. 2011; Johnson 2005; Lovejoy and Steuerwald 1995), little focus has been given to the positive factors associated with a diagnosis of BSD for youth, 
particularly positive schemas. Gaining a better understanding of not only the negative factors associated with BSD but also the positive factors (i.e., positive schemas) could ideally help with better identification of youth at risk for developing BSD and could provide information about protective factors that may buffer against the negative impacts of BSD. Positive schemas are also identifiable and potentially modifiable and may serve as an important intervention target for youth with psychopathology.

\section{The Parenting Context}

While BSD is largely influenced by genetics (Craddock and Jones 1996), environmental factors also have substantial etiological and phenomenological importance. The parenting context has been positioned as one of the most important environmental factors for understanding the onset and course of BSD (Belardinelli et al. 2008). Specifically, a parenting environment characterized by low levels of parental warmth or acceptance (e.g., excessive disapproval) and high levels of psychological control (e.g., promoting parental dependence) are generally related to BSD (Alloy et al. 2006; Geller et al. 2000, 2002; Geller et al. 2004).

Less considered is how parenting relates to the development of youths' positive functioning (e.g., resiliency, positive schemas). Resiliency is often regarded as being embedded in larger systems such as the parenting context (Patterson 2002; Masten 2007; Masten and Obradovic 2007). Caregivers have also been shown to play a vital role in prevention and intervention programs that promote resiliency (Draper et al. 2009; Prevatt 2003; Silk et al. 2007). Parenting is widely considered foundational for the development of schemas, and much research has shown a relation between the parenting context and the development of negative but not positive schemas (Garber and Flynn 2001; Hammen 1992; Harris and Curtin 2002; Kaslow et al. 1994; for an exception see Lumley et al. 2012). Based on the strong evidence for the relation between parenting, BSD, resiliency, and schema development, the current study aimed to understand the relation between parenting context and resiliency while considering positive schemas as a mediating factor, specifically for youth with BSD. Of practical importance, caregivers of youth with BSD could benefit from research that examines not only what aspects of parenting might be most problematic for youth, but also how aspects of parenting might be associated with youths' positive functioning (Schenkel, West, Harral, Patel, \& Pavuluri 2008).

\section{The Current Study}

Working within a positive clinical psychology framework, the current study was designed to enrich understanding of positive functioning for youth with BSD. Given the limited knowledge in this area, a qualitative component was included to gather both youth and caregiver perspectives about positive functioning for youth with BSD. This study also sought to understand how the parenting context relates to $\mathrm{BSD}$, shedding light on key parenting characteristics (i.e., acceptance and warmth, psychological control) that are associated with positive functioning for youth with BSD (i.e., resiliency, positive schemas, reduced psychopathology). Of particular significance, this 
study considered positive schemas as a potential mediator of the relation between parenting context and resiliency.

It was predicted that positive parenting strategies (i.e., acceptance) would be related to reduced levels of psychopathology (depression, mania), while negative parenting strategies (i.e., high parental psychological control) would be related to higher levels of psychopathology (depression, mania). Further, it was predicted that positive parenting strategies (i.e., acceptance) would be related to more positive schemas, which would then predict higher levels of youth resiliency. At the same time, it was predicted that negative parenting strategies (i.e., high parental psychological control) would be related to the development of fewer positive schemas, which would then predict lower levels of youth resiliency.

Given the differences in caregiver and youth reports of parenting (Boughton and Lumley 2011), models including both youth and caregiver reports of parenting were examined. Such information is relevant for informing environmental targets of intervention for this population.

\section{Method}

\section{Participants}

Participants consisted of 18 caregivers of female $(n=11)$ and male $(n=7)$ youth aged 8 to 18 years $(M=13.00, S D=3.01)$ who had received a diagnosis of Bipolar Spectrum Disorder (BSD) through a tertiary care facility. Of these families, 9 female and 4 male youth participated, resulting in 13 caregiver-child dyads. ${ }^{1}$ While undeniably a small sample, such a population is inherently difficult to recruit, and necessary statistical safeguards were implemented to reduce error and provide conservative estimates and results (see Analyses section). As such, this sample should be considered as a preliminary sample which may inform future research with this difficult-to-recruit yet important-to-study population.

Most caregivers were biological or adoptive mothers (88.9\%). For this sample, 16 guardians reported their youth taking psychotropic medications at the time of the study. All participants $(100 \% ; n=18)$ reported a biological family history of mental health difficulties and $61.1 \%(n=11)$ had a biological relative with a diagnosis of BSD. Reported family income was as follows: $16.7 \%$ earned below $\$ 20,000,33.3 \%$ earned between $\$ 20,000$ and $\$ 39,999,5.6 \%$ earned between $\$ 40,000$ and $\$ 59,999,11.1 \%$ earned between $\$ 60,000$ and $\$ 79,999$, and $33.3 \%$ earned $\$ 80,000$ or above.

\section{Measures}

Youth and caregiver perspectives of positive functioning A single question was provided at the end of a caregiver telephone interview to gather participants' experiences for qualitative exploration. In an open interview format, the caregiver was asked to tell the researcher "the best things about their child/youth". The caregiver's response was audio recorded and later transcribed. A single question was also given at the end of

\footnotetext{
${ }^{1}$ When available all data were used. This resulted in a sample size of 18 for analyses involving caregiver responses and a sample size of 13 for analyses involving youth or dyad responses.
} 
the youth interview to gather the youth's perspective. In an open interview format, the youth was asked to tell the researcher "the best things about him/herself." The youth's response was audio recorded and later transcribed.

Resiliency The Resiliency Scale (RS-14; Wagnild and Young 1993) was used to measure beliefs about one's ability to cope with adversity. Youth and caregivers completed the measure rating the youth's ability to cope. The scale comprises a global resiliency factor. Respondents indicated their rate of agreement with each statement using a 7-point Likert scale $(1=$ Strongly Disagree to $7=$ Strongly Agree $)$. For the current study the RS-14 evidenced high reliability when used with both caregiver $(\alpha=.93)$ and youth $(\alpha=.87)$ samples.

Positive schemas The Positive Schema Questionnaire (PSQ; Keyfitz et al. 2013) was used to measure youth's positive schemas. The scale comprises 20 items measuring: Worthiness, Self-Efficacy, Optimism, Success, and Interpersonal Trust. Youth indicated the extent to which they agreed with each statement using a 6-point Likert scale $(1=$ Completely untrue of me to $6=$ Describes me perfectly $)$. In the current study, an overall Positive Schema score was calculated by summing all items. The PSQ demonstrated high internal consistency $(\alpha=.96)$ for the current sample.

Mania symptoms The Child Mania Rating Scale (CMRS; Pavuluri et al. 2006) was used as a measure of youth mania symptoms. The measure is comprised of 21 items designed to reflect the DSM-IV criteria for a manic episode. Caregivers rated the items based on their youths' behaviour and emotions in the past month using a 4-point Likert scale $(0=$ Never/Rare to $3=$ Very Often $)$. The current study yielded good reliability $(\alpha=.92)$.

Depression symptoms The Beck Depression Inventory for Youth (BDI-Y; Beck et al. 2001) was used to measure depression symptoms. Caregivers and youth indicated the extent to which each statement described them/their youth based on a 4-point Likert scale $(0=$ Never to $3=$ Always $)$. The BDI-Y evidenced high internal consistency for both caregiver $(\alpha=.93)$ and youth $(\alpha=.89)$ reporting.

Parenting The Child Report of Parenting Behavior Inventory- Short form (CRPBI; Schludermann and Schludermann 1988) was used to provide a measure of both caregiver-and youth-reported parenting attributes. The Acceptance and Psychological Control subscales were used from the shortened version of the CRPBI. Youth and caregivers were asked to rate characteristics about their caregiver/self on a 5-point Likert scale $(1=$ Disagree to $5=$ Agree $)$. For the current study, the caregiver report measure yielded moderate reliability for the two subscales ( $\alpha=.73$ and $\alpha=.69$, respectively) and the youth report measure yielded high reliability ( $\alpha=.82$ and $\alpha=.90$, respectively).

\section{Procedure}

Caregivers of youth who received a diagnosis of BSD following a comprehensive assessment at a tertiary care facility were contacted by telephone and provided with 
information about the current study and a toll-free number for the research lab conducting the study. When a participant contacted the toll-free number, oral consent was obtained and the caregiver was asked to complete the caregiver questionnaires and single qualitative question described in the Measures section. After oral assent was obtained, the youth was asked to complete the youth questionnaires and single qualitative question described in the Measures section. Following participation, a gift card was mailed to the family to thank them for taking part in the study. This study was approved by the local ethics Review Board.

\section{Data Analysis}

\section{Qualitative Analyses}

To explore positive qualities of youth with BSD, the guidelines for examining qualitative data suggested by Braun and Clarke (2006) were adapted and applied to the qualitative question described in the Measures section. Rather than approaching the analyses with particular themes in mind, the researcher (first Author) used a grounded theory approach to conduct a thematic analysis and look to participant responses for salient themes.

In the first step of data analysis, the data gathered from the specific qualitative questions (caregiver and youth) were transcribed so the researcher could become more familiarized with the data. The data were then checked again for accuracy by a secondary researcher. Next, the researcher generated a list of potential codes and unique features of the data set by reviewing an inclusive and comprehensive portion of the transcripts $(>75 \%)$. Codes were developed until saturation (i.e., no new codes were found). Following this, the researcher collated the codes and features to develop themes. A theme was considered as any pattern of results that represented a unique meaning or idea within the data. Themes were developed based on their relevance to the question at hand (i.e., introducing a new idea related to the "best things" about the youth), not based on the prevalence of the specific meaning or idea. Next, the created themes were checked against the full data set for further review and to verify that they provided an adequate fit for the data. Finally, the themes were refined and the final coding scheme was developed. The coding scheme was then used to examine the data to determine the frequency of the established themes.

After the completion of the thematic scheme, all transcripts were coded. With regards to examining the qualitative information, prevalence of theme, as outlined by the coding scheme, was counted as the number of participants who articulated the theme across the entire data set. The replicability of the coding scheme and specific themes was established by having a second researcher code the two questions based on the established coding scheme. A high level of inter-rater reliability was observed (100\% agreement).

\section{Quantitative Analyses}

In the current study, Positive Schemas were proposed as a possible mediator of the relation between Parenting Characteristics and Resiliency in youth with a BSD. To 
explore this, a series of univariate mediation analyses were conducted. Separate mediation models were fitted for youth-and caregiver-reported parental Acceptance and Psychological Control predicting youth-reported Resiliency, resulting in four models.

For the univariate mediation models tested, youth and caregiver reports of Parenting Characteristics (i.e., Acceptance and Psychological Control; CRPBI) were the independent variables, youth-reported Resiliency (RS) was the dependent variable, and youth-reported Positive Schema total score (PSQ) was used as the mediator in each analysis.

For all models tested, the indirect effect was estimated using the non-parametric bootstrapping procedure described by Preacher and Hayes (2004). This approach is widely regarded as an improvement to Baron and Kenny's (1986) causal steps mediation method and is a suitable choice when sample size is small (Hayes 2009). The bootstrapping procedure yields more accurate estimates of confidence intervals for the indirect effect than the Sobel test, and was carried out using the SOBEL macro for SPSS (Preacher and Hayes 2008). The indirect effect was bootstrapped with 5000 sample indirect effects (with replacement) using the current sample as the population of possible indirect effects. This yielded a point estimate of the population value of the indirect effect (unstandardized regression coefficient), its standard error, and 95\% confidence interval (CI).

A post hoc power analysis, conducted using G*Power 3 (Faul et al. 1996), showed there was a $14 \%$ chance of detecting a medium sized effect $\left(f^{2}=0.15\right)$ and a $28 \%$ chance of detecting a large effect $\left(f^{2}=0.35\right)$ for a regression model including three predictor variables (i.e., parenting, positive schemas, and the interaction term) based on the sample size of 13 participants. With regard to this small sample size and low level of power, Preacher and Hayes (2004) outline that the bootstrapping process is not based on "large-sample" theory and thus can be applied to small samples with relative confidence. Consequently, this analytical approach was used while also acknowledging the limitations inherent to this small, difficult to obtain sample.

\section{Results}

\section{Qualitative Results}

Analysis of caregiver and youth responses revealed four key themes with multiple subcategories ( $n=11$ and $n=5$, respectively; see Table 1, Fig. 1, and Fig. 2). In total, 76 caregiver comments and 26 youth comments were provided in response to the single qualitative questions. Overall, $92.1 \%$ of the caregiver data and $100 \%$ of the youth data were allocated to a theme.

Caregiver perspective The themes present when caregivers were asked, "what are the best things about your child", varied greatly (Fig. 3). All caregiver comments were coded for the presence of the identified themes, not the frequency with which they appeared within any one transcript. All percentages presented are based on the occurrence of the thematic response in relation to the total number of responses. Only a few 
Table 1 Coding Scheme for Parental and Youth Responses

\begin{tabular}{|c|c|c|}
\hline Main Themes & Subcategories & Sample \\
\hline \multicolumn{3}{|l|}{ Parent Responses } \\
\hline $\begin{array}{l}\text { 1. Interpersonal } \\
\text { Relationships }\end{array}$ & $\begin{array}{l}\text { Flexibility } \\
\text { Assisting Others } \\
\text { Positive Relationship } \\
\quad \text { Qualities }\end{array}$ & $\begin{array}{l}\text { "amenable to suggestions" } \\
\text { "passion for helping others" } \\
\text { "loving" }\end{array}$ \\
\hline 2. Internal Characteristics & $\begin{array}{l}\text { Energy } \\
\text { Humour } \\
\text { Creativity } \\
\text { Intelligence }\end{array}$ & $\begin{array}{l}\text { "energetic" } \\
\text { "sense of humour" } \\
\text { "thinks outside the box" } \\
\text { "smart" }\end{array}$ \\
\hline 3. External Characteristics & $\begin{array}{l}\text { Interests/Hobbies } \\
\text { World View }\end{array}$ & $\begin{array}{l}\text { "good hockey player" } \\
\text { "unique way of looking at the world" }\end{array}$ \\
\hline 4. Agency & $\begin{array}{l}\text { Self Advocates } \\
\text { Determination/Commitment }\end{array}$ & $\begin{array}{l}\text { "defends himself" } \\
\text { "her drive" }\end{array}$ \\
\hline \multicolumn{3}{|l|}{ Youth Responses } \\
\hline $\begin{array}{l}\text { 1. Interpersonal } \\
\text { Relationships }\end{array}$ & $\begin{array}{l}\text { Assisting Others } \\
\text { Positive Relationship } \\
\text { Qualities }\end{array}$ & $\begin{array}{l}\text { "determined to help others" } \\
\text { "good friend" }\end{array}$ \\
\hline 2. Internal Characteristics & $\begin{array}{l}\text { Humour } \\
\text { Creativity } \\
\text { Intelligence }\end{array}$ & $\begin{array}{l}\text { "humour-my ability to make light of } \\
\text { situations" } \\
\text { "creative" } \\
\text { "fast learner" }\end{array}$ \\
\hline 3. Interests/Hobbies & & "ability to work with engines" \\
\hline $\begin{array}{l}4 . \\
\text { Determination/- } \\
\text { Commitment }\end{array}$ & & "hard worker" \\
\hline
\end{tabular}

comments were not coded due to a lack of fit with any specific category ( $n=5$; e.g., "has an innocence about him"). Based on the four main themes, the most frequent comments were made in the Interpersonal Relationships (i.e., qualities important to fostering positive interpersonal relationships; e.g., "likeable", "loyal", etc.) and Internal Characteristics (e.g., "thinking outside the box", "being very creative", etc.) domains (35.85\% each), followed by External Characteristics (15.09\%; e.g., "unique way of

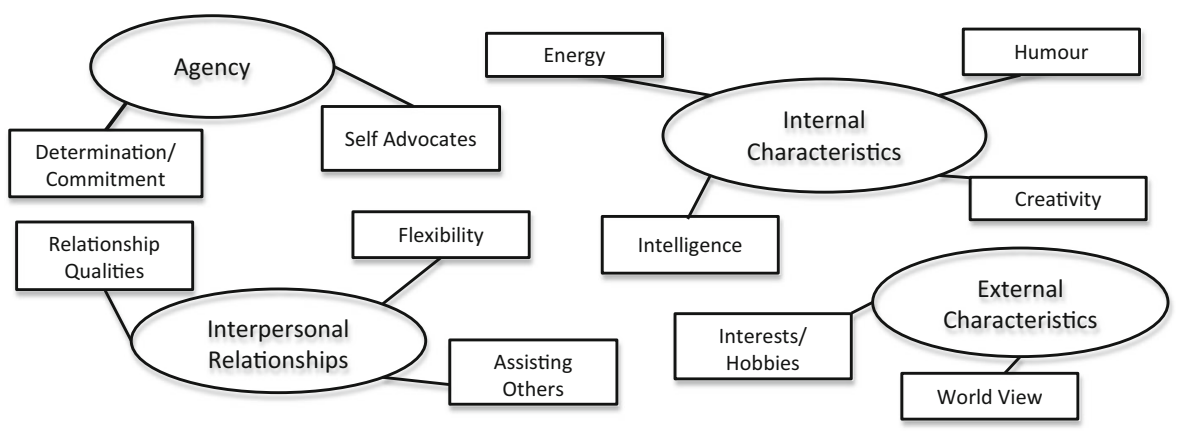

Fig. 1 Thematic map outlining the main qualitative themes and subcategories for the caregiver's perspective of the "best things" about their youth 


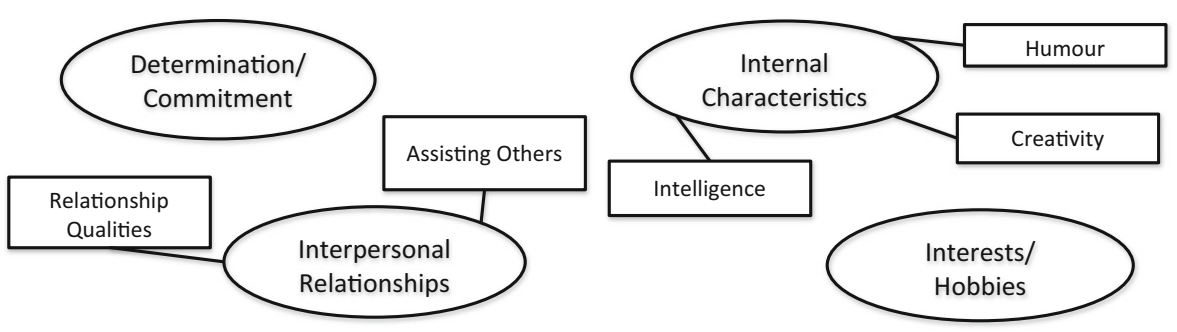

Fig. 2 Thematic map outlining the main qualitative themes and subcategories for the youth's perspective of the "best things" about themselves

looking at things in the world", "he is an excellent athlete", etc.), and Agency (13.21\%; e.g., "dedicated", "committed", "motivated", etc.).

Youth perspective The themes present when youth were asked, "what are the best things about you", also varied greatly (Fig. 4). All youth comments were coded for the presence of the identified themes not the frequency with which they appeared within any one transcript. Based on the four main themes, the most frequent comments were made in the Interpersonal Relationships domain (55\%; e.g., "caring", "sociable", etc.), followed by External Characteristics (25\%; e.g., "I can play sports" "I like reading",

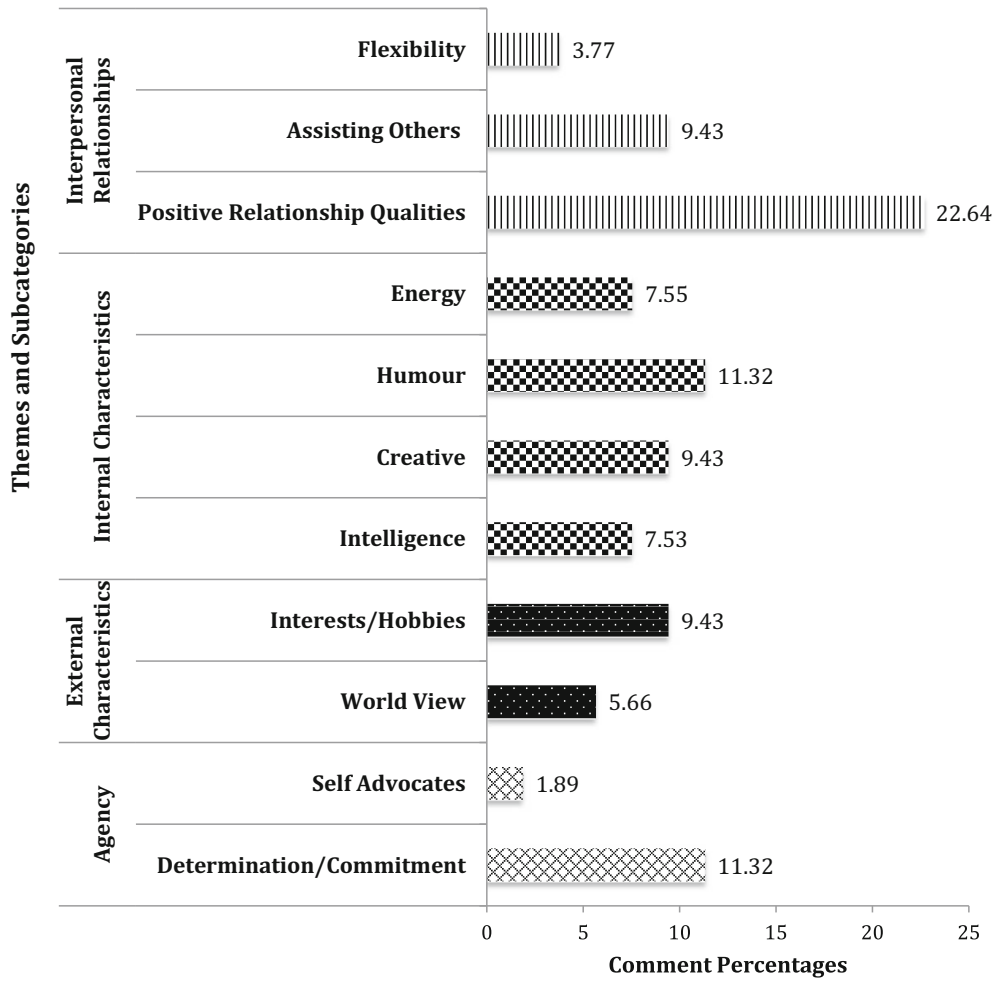

Fig. 3 Themes and subcategories for caregiver comments regarding the "best things" about their youth $(n=53)$, presented in percentages 


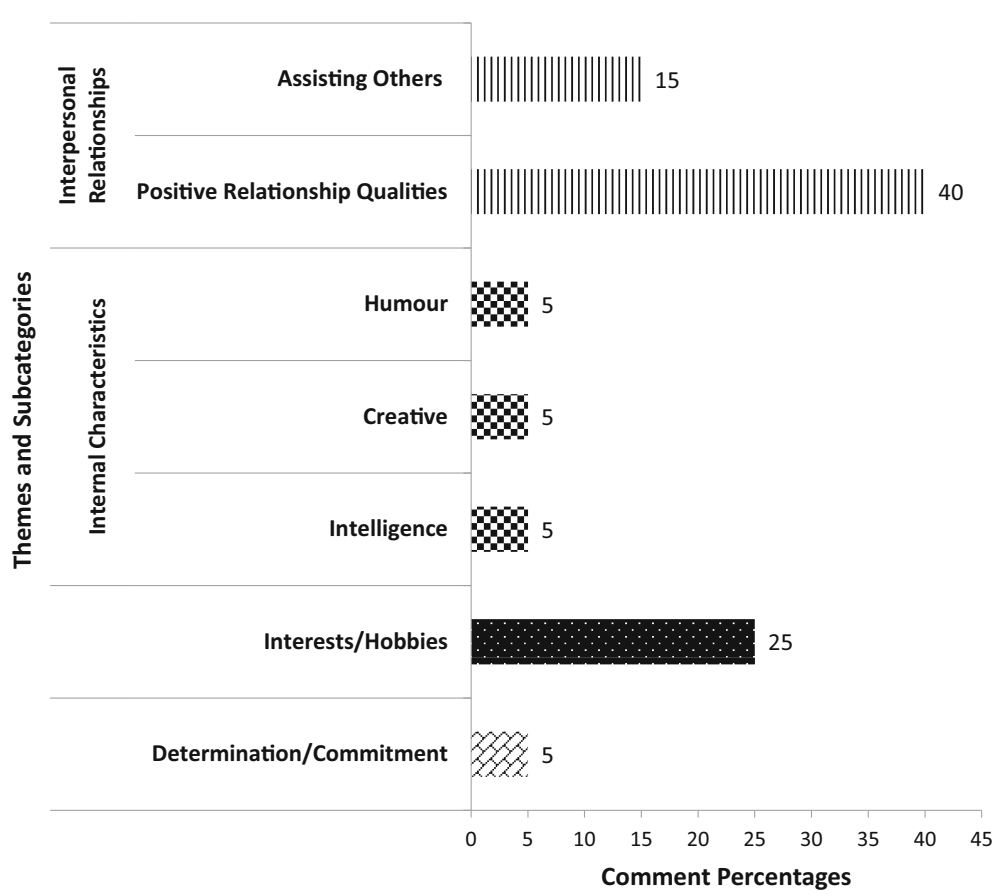

Fig. 4 Themes and subcategories for youth comments regarding the "best things" about themselves $(n=20)$, presented in percentages

etc.), Internal Characteristics (15\%; e.g., "creative ways", "fast learner", etc.), and Agency (5\%; e.g., "I am a hard worker").

\section{Descriptive Statistics}

The means, standard deviations and zero-order correlations for the CRPBI, RS, PSQ, CMRS, and BDI-Y, can be found in Table 2.

\section{Caregiver Report of Parenting}

Caregiver-reported parental Acceptance (CRPBI) was significantly and positively related to youth- and caregiver-reported Resiliency (RS), and Positive Schemas (PSQ). Caregiver-reported parental Acceptance (CRPBI) was also significantly and negatively related to youth-reported Depression (BDI-Y). Caregiver-reported parental Psychological Control (CRPBI) was significantly and positively related to caregiverreported Resiliency (RS).

\section{Youth Report of Parenting}

Regarding youth-reported parenting, based on the CRPBI, youth-reported parental Acceptance was significantly and positively related to caregiver-reported parental Acceptance (CRPBI), youth-and caregiver-reported Resiliency (RS), and Positive 
Table 2 Descriptive Statistics

\begin{tabular}{|c|c|c|c|c|c|c|c|c|c|c|c|c|}
\hline Measure $^{1}$ & $M$ & $S D$ & 1. & 2. & 3. & 4. & 5. & 6. & 7. & 8. & 9. & 10. \\
\hline \multicolumn{13}{|c|}{ CRPBI-Youth Version } \\
\hline 1. Acceptance & 44.39 & 5.24 & & .33 & $.70 * *$ & .21 & $.70 * *$ & $.58 *$ & $.81 * *$ & -.01 & -.45 & -.35 \\
\hline $\begin{array}{l}\text { 2. Psychological } \\
\text { Control }\end{array}$ & 30.08 & 8.94 & & 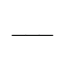 & .06 & $.47 *$ & .09 & .45 & .10 & -.35 & -.09 & -.33 \\
\hline \multicolumn{13}{|c|}{ CRPBI-Parent Version } \\
\hline 3. Acceptance & 45.61 & 3.60 & & & - & .10 & $.53 *$ & $.50 *$ & $.61 *$ & -.11 & $-.65 * *$ & -.06 \\
\hline $\begin{array}{l}\text { 4. Psychological } \\
\text { Control }\end{array}$ & 19.11 & 4.36 & & & & - & -.27 & $.46^{*}$ & -.13 & -.33 & .05 & -.28 \\
\hline \multicolumn{13}{|l|}{ RS-Youth Version } \\
\hline $\begin{array}{l}\text { 5. Resilience } \\
\text { Total }\end{array}$ & 70.77 & 15.19 & & & & & $\underline{-}$ & .44 & $.86^{* *}$ & -.06 & $-.65^{* *}$ & -.49 \\
\hline \multicolumn{13}{|l|}{ RS-Parent Version } \\
\hline $\begin{array}{l}\text { 6. Resilience } \\
\text { Total }\end{array}$ & 57.56 & 17.20 & & & & & & - & .19 & $-.64 * *$ & -.35 & $-60 * *$ \\
\hline \multicolumn{13}{|l|}{ PSQ } \\
\hline $\begin{array}{l}\text { 7. Positive } \\
\text { Schema Total }\end{array}$ & 86.62 & 21.92 & & & & & & & 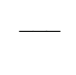 & .29 & $-.60 *$ & -.21 \\
\hline \multicolumn{13}{|l|}{ CMRS } \\
\hline 8. Mania Total & 21.17 & 11.74 & & & & & & & & - & .02 & $.64 * *$ \\
\hline \multicolumn{13}{|l|}{ BDI-Y-Youth Version } \\
\hline $\begin{array}{l}\text { 9. Depression } \\
\text { Total }\end{array}$ & 19.46 & 9.43 & & & & & & & & & 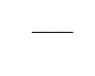 & $.63 *$ \\
\hline \multicolumn{13}{|c|}{ BDI-Y-Parent Version } \\
\hline $\begin{array}{l}\text { 10. Depression } \\
\text { Total }\end{array}$ & 18.22 & 10.38 & & & & & & & & & & \\
\hline
\end{tabular}

Note. $* p<.05, * * p<.001$, one-tailed

1 CRPBI = Child Report of Parenting Behaviour; RS = Resilience Scale; PSQ = Positive Schema Questionnaire; CMRS = Child Mania Rating Scale; BDI-Y = Beck Depression Inventory - Youth

Schemas (PSQ). Youth-reported parental Psychological Control (CRPBI) was significantly and positively related to caregiver-reported parental Psychological Control (CRPBI).

\section{Positive Schema Mediation.}

Acceptance The first model examined Positive Schemas (PSQ) as a mediator between youth-reported parental Acceptance (CRPBI) and youth-reported Resiliency (RS). The overall model was significant $[F(2,10)=13.54, p=.001]$ and accounted for $73 \%$ of the variance in predicting youth-reported Resiliency. The indirect effect of Positive Schemas was significant at $p=.013$. Bootstrapping results confirmed that the direct effect of youth-reported parental Acceptance on youth-reported Resiliency was much smaller and not significant when the mediation path was included. Table 3 outlines the confidence intervals for the indirect effect. Path estimates for each pathway (see Fig. 5) suggested increased youth-reported parental Acceptance lead to increased Positive Schemas, which in turn lead to increased youth-reported Resiliency. 
Table 3 Bootstrapped Point Estimates (B), Standard Error (SE) and Confidence Intervals (CIs) for the Indirect Effect of Parenting on Resilience with Positive Schemas as a Mediator

\begin{tabular}{llll}
\hline Model Summary & $B$ & $S E$ & $95 \%$ CI \\
& Lower Upper
\end{tabular}

\section{Model 1}

Youth reported parental Acceptance on youth reported Resilience

$\begin{array}{rrrr}2.00 * & .87 & .72 & 3.99 \\ 2.32 & 3.94 & -.76 & 4.69 \\ .14 & .51 & -1.14 & .79\end{array}$

Youth reported parental Psychological Control on youth reported Resilience

\section{Model 4}

Caregiver reported parental Psychological Control on youth reported Resilience

Note. * indicates significant at $p<.05 .95 \%$ CI Estimates based on 5000 bootstrapped sample

Next, Positive Schemas (PSQ) were examined as a potential mediator between caregiver-reported parental Acceptance (CRPBI) and youth-reported Resiliency (RS). The overall model was significant $[F(2,10)=13.54, p=.001]$ and accounted for $73 \%$ of the variance in predicting youth-reported Resiliency. The indirect effect of Positive Schemas was significant, $p=.033$. However, the bootstrapped results showed that the direct effect of youth-reported parental Acceptance on caregiver-reported Resiliency did not significantly differ when the mediation path was included (see Table 3).

Psychological control The third model examined Positive Schemas (PSQ) as a potential mediator between youth-reported parental Psychological Control and youth-

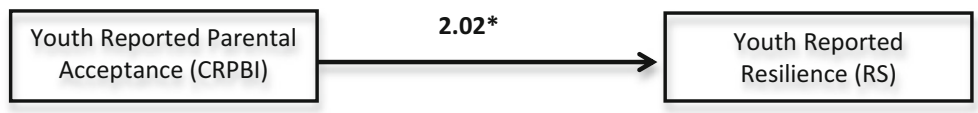

(a)

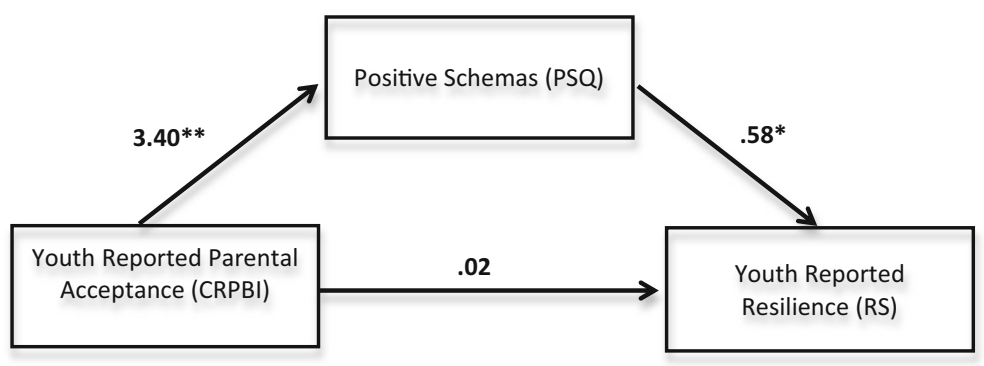

(b)

Fig. 5 Mediation model of youth reported parental acceptance predicting (a) path estimates for the direct effect of youth reported parental acceptance on youth reported resilience and (b) path estimates for the indirect effect of youth reported parental acceptance on youth reported resilience $* p<.05, * * p<.001$. 
reported Resiliency (RS). The indirect effect of Positive Schemas was not significant, $p=.754$ (see Table 3). Finally, Positive Schemas (PSQ) was examined as a potential mediator between caregiver-reported parental Psychological Control (CRPBI) and youth-reported Resiliency (RS). The indirect effect of Positive Schemas was not significant, $p=.915$ (see Table 3).

\section{Discussion}

Youth with BSD comprise a unique subset of adolescents experiencing psychopathology (i.e., exhibiting a mix of depressive and manic symptomatology), and little is known about positive functioning and whether parenting context variables relate to aspects of positive functioning for youth with this diagnosis. The goal of this study was to gain a better understanding of factors relating to positive functioning for youth with a diagnosis of BSD. In line with recent recommendations, a mixedmethods approach was used in order to foster a more open, comprehensive understanding of positive functioning among these youth, rather than solely providing caregivers and youth with predetermined descriptive scales (Armstrong et al. 2005; Braun and Clarke 2006; Krahn et al. 1995). In addition to a qualitative component, this study also quantitatively explored the relation between parenting context and positive functioning (resiliency, positive schemas) for youth with a diagnosis of BSD. Results highlight caregivers and youth are able to reflect on and articulate several aspects of positive functioning despite the severity of a diagnosis such as BSD, particularly in the domain of interpersonal relationship qualities and internal characteristics. As well, this study provided evidence for the relation between parenting context and positive functioning for youth with BSD, and results further suggest positive schemas as one mechanism that may explain the relation between parenting and positive outcomes for these youth.

\section{Qualitative Findings}

The qualitative analysis revealed most caregivers described the "best thing" about their youth as being related to interpersonal relationship qualities (e.g., being friendly, loving, likeable, etc.) or aspects of their youth's internal characteristics (e.g., humour, being smart, creative, etc.). This finding is consistent with a previous qualitative study with adults diagnosed with BSD, in which they described themselves as possessing amplified internal states and more human connectedness (Lobban et al. 2012). Youth responses about the "best things" about themselves cohered largely with caregiver responses, with youth speaking about their own interpersonal relationship qualities (e.g., caring, sociable, etc.).

This is an interesting finding given much research has focused on the differences between parent and youth reporting (Boughton and Lumley 2011; Clay et al. 2008; De Los Reyes and Kazdin 2005; Kolko and Kazdin 1993). However, much of the research finding differences between caregiver and youth reporting has focused on negative outcomes (e.g., family conflict, family stress, parental dysfunction; see Boughton and Lumley 2011, for a review), while in contrast the current study focused on measuring 
caregiver and youth perspectives of positive outcomes. This difference is worth exploring in future research to determine if this finding is unique to our sample (i.e., youth with a diagnosis of BSD) or specific to reports of positive functioning as compared to negative outcomes. As well, to our knowledge this is the first study to qualitatively examine youth and caregiver impressions of positive functioning in the context of BSD, and future studies should endeavour to include such mixed methods approaches to potentially replicate these findings and provide a richer understanding of living with this complex diagnosis.

\section{Youth Report of Parenting}

Both youth report of parental acceptance and psychological control were significantly related to caregiver report of parental acceptance and psychological control, indicating general agreement on the parenting context. Although reporting was similar, the unique perspectives of both caregivers and youth in understanding the parenting context should not be underestimated. Including both caregiver and youth reports of parenting in the current study bolstered the inclusive nature of this study's methodological design.

Consistent with a large body of work highlighting the relation between parental acceptance and psychopathology (Geller et al. 2000, 2002, 2004; Parker 1983; Schenkel et al. 2008), higher levels of perceived parental acceptance were related to lower levels of reported depression in this sample. Further and consistent with hypotheses, youth-reported parental acceptance was significantly related to all aspects of positive functioning in this sample, including resiliency and positive schemas. These results provide corroborating evidence underscoring the importance of parental acceptance in the parent-child relationship for youth with BSD (Geller et al. 2000, 2004; Neeren et al. 2008; Rosenfarb et al. 1994) and add to this literature by considering how these important parenting characteristics relate to positive outcomes for youth with BSD. This finding may suggest that youth who see their caregivers as being warm and accepting may receive positive messages from their caregivers, and/or experience a positive parent-child relationship, which, in turn, leads youth to internalize and generalize such messages in the form of positive schemas. This synergistic process may then set the stage for positive outcomes including resiliency. These findings highlight the need for future research to continue to elaborate on mechanisms underlying the relation between parental acceptance and indicators of positive functioning for youth with a diagnosis of BSD.

Interestingly, youth-reported parental psychological control was not significantly related to any positive functioning outcomes. Previous research has suggested a relation between parental psychological control and greater levels of psychopathology for youth with BSD (Geller et al. 2000, 2002; Parker 1983; Schenkel et al. 2008), thus these findings contradicted hypotheses. Much of the previous research examining parental psychological control has focused on the presence of psychological control prior to a diagnosis of BSD and has implied parental psychological control is a negative factor present prior to episodes of depression and/or mania (Neeren et al. 2008). Yet, research has not examined the relation between parental psychological control and positive outcomes for youth with BSD. In light of our findings, it seems possible that positive parenting facets, such as warmth and acceptance, are more etiologically and phenomenologically important in understanding positive outcomes for youth with BSD than 
negative parenting characteristics such as psychological control. Additional research and replication with larger and more diverse samples is needed to confirm such musings.

\section{Caregiver Report of Parenting}

Consistent with the aforementioned youth results, caregiver-reported parental acceptance was significantly related to lower levels of youth-reported depression. Gathering further support for hypotheses and consistent with youth report of parenting, caregiverreported parental acceptance was also significantly related to all aspects of positive functioning (i.e., resiliency, positive schemas).

Contrary to initial hypotheses, caregiver-reported parental psychological control was significantly and positively related to caregiver-reported youth resiliency. This finding is surprising given research indicating parental psychological control is generally related to negative outcomes for youth with BSD (Geller et al. 2000, 2002; Parker 1983; Schenkel et al. 2008). It is common for youth with this diagnosis to have mixed emotional states (i.e., depression, mania), making parenting youth with BSD a difficult task (Pavuluri et al. 2004; Pavuluri 2008). It is possible that a caregiver higher in parental psychological control may help a youth who has difficulty with manic and depressive states by providing a firm structure for how the youth should be thinking about themselves, the world, and the future. Yet, this association was found within caregiver report only and is speculative. There may be other explanations for this pattern in these data including parents own mental health status, biased perceptions or the influence of measurement error within a small sample. Future research should aim to explore such reflections and replicate these associations within a larger sample.

\section{Positive Schema Mediation}

After exploring the relations between parenting and positive outcomes for youth with $\mathrm{BSD}$, the next step was to determine if positive schemas act as a mediator for the relation between parenting context and resiliency. It was hypothesized that positive schemas would mediate the relation between parental acceptance and resiliency as well as between parental psychological control and resiliency. Overall, four models were tested including caregiver and youth-reported parental acceptance and psychological control as the main parenting characteristics, predicting youth-reported resiliency, while considering positive schemas as a mediator.

Consistent with hypotheses, positive schemas acted as a significant mediator between youth-reported parental acceptance and youth-reported resiliency. Although cross-sectional in nature, this analysis is consistent with a model suggesting increased levels of youth perceived parental acceptance may lead to increased development of positive schemas, which in turn result in higher levels of youth perceived resiliency. This significant mediation model accounted for a large amount of the variance in predicting youth-reported resiliency and seems to be a robust finding given the small sample size for this study. Consistent with previous findings (Keyfitz et al. 2013), but extended to youth with BSD, the development of positive schemas seems to play an important role when predicting resiliency outcomes. The current study also added novel information to the field of positive psychology by examining not only relations 
between parenting characteristics and resiliency, but also considering the associations between parenting characteristics and positive schemas which in turn may promote resiliency. Finally, this model sheds light on this relation specifically for youth with $\mathrm{BSD}$, suggesting that despite the difficulties associated with a diagnosis of BSD, positive trajectories from positive parenting characteristic through positive core schemas to resiliency may also be unfolding.

Contrary to hypotheses, the second mediation model did not support positive schemas as a mediator between caregiver-reported parental acceptance and youthreported resiliency. It is interesting to note the overall model was significant, suggesting that caregiver-reported parental acceptance and positive schemas do predict resiliency in youth with BSD, but potentially not within a mediation framework. Consistent with previous research underscoring youths' perceptions as central (De Los Reyes and Kazdin 2005), the lack of significant mediation in this model may suggest that the youths' (versus caregivers') perceptions of parenting may be most influential on the development of positive schemas, which in turn promotes resiliency. It may also be that this analysis was underpowered, particularly for work examining these constructs across multiple informants.

The remaining models tested positive schemas as a mediator between youth-or caregiver-reported parental psychological control and youth-reported resiliency. Contrary to hypotheses, neither overall model was significant, suggesting parental psychological control and positive schemas together do not explain significant variance in resiliency, regardless of who reports on the psychological control.

Research examining the relation between negative parenting strategies and the development of positive schemas is lacking. Nonetheless, it was suspected that negative parenting would be associated with the development of fewer positive schemas, which in turn would be associated with lower levels of resiliency. However, this relation was not observed in the present study. Theorists have suggested the influence of negative parenting characteristics (e.g., parental rejection) may be more salient than parental warmth and acceptance in the development of negative schemas (Garber and Flynn 2001; Kaslow et al. 1994). Based on the findings from the current study, it is possible that parental acceptance, in turn, may be more salient in the development of positive schemas, than negative parenting characteristics (e.g., criticism, psychological control) are in the lack of development of positive schemas. It is also important to consider that the relation between parental psychological control and positive outcomes for these youth with BSD may be complex given the univariate relations to youth resiliency and thus may not have interacted with positive schemas and resiliency in a predictable way.

\section{Limitations and Future Directions}

The results of the current study are necessarily preliminary with regard to the small sample size, and must be interpreted with caution. Replication with a larger sample of male and female youth with a diagnosis of BSD would be ideal, keeping in mind that it is difficult to recruit this specialized clinical sample. Although the sample is small, this study does shed light on some important and novel relations and can help guide decisions for more extensive clinical studies.

This study used subjective reporting for a number of the central variables measured. Subject reporting may have resulted in reporting bias (e.g., social desirability, 
defensiveness, etc.). The multi-informant design of this study was used to address some of these concerns, however additional studies including, for example, more in-depth interviews, physiological research, and/or home observations would provide further validation of these constructs.

Lastly, this study utilized cross-sectional data limiting the interpretation of findings. When utilizing cross-sectional designs, it is difficult to determine cause and effect. In particular, the significant mediation analysis suggesting that positive schemas mediate the relation between parental acceptance and resiliency would be strengthened if explored using longitudinal methods. However, the mediational model tested in the current study is consistent with theory and previous research suggesting parenting relates to schema development, which influences youth functioning.

\section{Clinical Implications}

It has been suggested that clinicians generally do not attend to enhancing the positive aspects associated with a diagnosis of BSD, and that treatment of BSD symptoms may result in less benefit for individuals with BSD when positive aspects of certain symptoms of BSD are not recognized (Galvez et al. 2011). Overall, gaining a better understanding of not only the negative aspects identified by youth with BSD but also the positive aspects could provide information about protective factors that may buffer against the negative impacts of the illness. This study also highlights positive parenting context and schemas in particular, both of which may be identified, targeted and potentially modified when intervening with youth and their families.

\section{Compliance with Ethical Standards}

Ethical Approval All procedures performed in studies involving human participants were in accordance with the ethical standards of the institutional and/or national research committee and with the 1964 Helsinki declaration and its later amendments or comparable ethical standards.

Conflict of Interest On behalf of all authors, the corresponding author states that there is no conflict of interest.

\section{References}

Adler, N. E., Horowitz, M., Garcia, A., \& Moyer, A. (1998). Additional validation of a scale to assess positive states of mind. Psychosomatic Medicine, 60(1), 26-32.

Alloy, L. B., Abramson, L. Y., Smith, J. M., Gibb, B. E., \& Neeren, A. M. (2006). Role of parenting and maltreatment histories in unipolar and bipolar mood disorders: Mediation by cognitive vulnerability to depression. Clinical Child and Family Psychology Review, 9(1), 23-64.

Alloy, L. B., Nusslock, R., \& Boland, E. M. (2015). The development and course of bipolar spectrum disorders: An integrated reward and circadian rhythm dysregulation model. Annual Review of Clinical Psychology, 11, 213-250.

Armstrong, M. I., Birnie-Lefcovitch, S., \& Ungar, M. T. (2005). Pathways between social support, family well being, quality of parenting, and child resilience: What we know. Journal of Child and Family Studies, $14(2), 269-281$. 
Baron, R. M., \& Kenny, D. A. (1986). The moderator-mediator variable distinction in social psychological research: Conceptual, strategic, and statistical considerations. Journal of Personality and Social Psychology, 51(6), 1173-1182.

Beck, J. S., Beck, A. T., \& Jolly, J. (2001). Beck Youth Inventories. San Antonio, TX: The Psychological Corporation.

Belardinelli, C., Hatch, J. P., Olvera, R. L., Fonseca, M., Caetano, S., Nicoletti, M., et al. (2008). Family environment patterns in families with bipolar children. Journal of Affective Disorders, 107(1-3), 299305.

Biederman, J., Faraone, S. V., Wozniak, J., Mick, E., Kwon, A., Cayton, G. A., \& Clarke, S. V. (2005). Clinical correlates of bipolar disorder in a large referred sample of children and adolescents. Journal of Psychiatric Research, 39(6), 611-622.

Boughton, K. L., \& Lumley, M. N. (2011). Parent prediction of child mood and emotional resilience: The role of parental responsiveness and psychological control. Depression Research and Treatment, 2011, 1-9.

Braun, V., \& Clarke, V. (2006). Using thematic analysis in psychology. Qualitative Research in Psychology, 3(2), 77-101.

Cacioppo, J. T., Gardner, W. L., \& Berntson, G. G. (1999). The affect system has parallel and integrative processing components: Form follows function. Journal of Personality and Social Psychology, 76(5), 839-855.

Clay, H., Surgenor, L. J., \& Frampton, C. M. (2008). Assessing emotional and behavioural problems in children: Factors associated with multiple informant consistency in New Zealand. New Zealand Journal of Psychology, 37(1), 10-16.

Cole, D. A., \& Turner, J. E. (1993). Models of cognitive mediation and moderation in child depression. Journal of Abnormal Psychology, 102(2), 271-281.

Craddock, N., \& Jones, I. (1996). Genetics of bipolar disorder. Journal of Medical Genetics, 36(8), 585-594.

Crowe, M., Inder, M., Carlyle, D., Wilson, L., Whitehead, L., Panckhurst, A., et al. (2012). Feeling out of control: A qualitative analysis of the impact of bipolar disorder. Journal of Psychiatric and Mental Health Nursing, 19(4), 294-302.

De Los Reyes, A. L., \& Kazdin, A. E. (2005). Informant discrepancies in the assessment of childhood psychopathology: A critical review, theoretical framework, and recommendations for further study. Psychological Bulletin, 131(4), 483-509.

Draper, K., Siegel, C., White, J., Solis, C. M., \& Faye, M. (2009). Preschoolers, parents, and teachers (PPT): A preventative intervention within an at risk population. Journal of Group Psychotherapy, 59(2), 221-242.

Duckworth, A. L., Steen, T. A., \& Seligman, M. E. (2005). Positive psychology in clinical practice. Annual Review of Clinical Psychology, 1, 629-651.

Faul, F., Erdfelder, E., Lang, A. G., \& Buchner, A. (1996). G*Power 3: A flexible statistical analysis program for the social, behavioral, and biomedical sciences. Behavioral Research Methods, 39(2), 175-191.

Fleming, J., \& Darley, J. M. (1986). Perceiving intention in constrained behaviour: The role of purposeful and constrained action cues in correspondence bias effects. Princeton, NJ: Princeton University Unpublished manuscript.Please check provided publisher location of this reference if correct.- $\rightarrow$

Fletch, K., Parker, G., \& Manicavasager, V. (2013). A qualitative investigation of hypomania and depression in Bipolar II Disorder. Psychiatry Quarterly, 84(4), 455-474.

Folkman, S. (1997). Positive psychological states and coping with severe stress. Science and Medicine, 45(8), 1207-1221.

Galvez, J. F., Thommi, S., \& Ghaemi, S. N. (2011). Positive aspects of mental illness: A review in bipolar disorder. Journal of Affective Disorders, 128(3), 185-190.

Garber, J., \& Flynn, C. (2001). Vulnerability to depression in childhood and adolescence. In R. E. Ingram \& J. M. Price (Eds.), Vulnerability to psychopathology: Risk across the lifespan (pp. 175-225). New York, NY: The Guilford Press.

Geller, B., Bolhofner, K., Craney, J. L., Williams, M., Delbello, M. P., \& Gundersen, K. (2000). Psychosocial functioning in prepubertal and early adolescent bipolar disorder phenotype. Journal of the American Academy of Child and Adolescent Psychiatry, 39(12), 1543-1548.

Geller, B., Craney, J. L., Bolhofner, K., Nickelsburg, M. J., Williams, M., \& Zimerman, B. (2002). Two-year prospective follow-up of children with a prepubertal and early bipolar disorder phenotype. American Journal of Psychiatry, 159(6), 927-933.

Geller, B., \& Luby, J. (1997). Child and adolescent bipolar disorder: A review of the past 10 years. Journal of the American Academy of Child and Adolescent Psychiatry, 36(9), 1168-1176.

Geller, B., Tillman, R., Craney, J. L., \& Bolhofner, K. (2004). Four-year prospective outcome and natural history of mania in children with a prepubertal and early adolescent bipolar disorder phenotype. Archives of General Psychiatry, 61(5), 459-467. 
Greenwald, A. G. (1980). The totalitarian ego: Fabrication and revision of personal history. American Psychologist, 35(7), 603-618.

Hammen, C. L. (1992). Cognitive, life stress, and interpersonal approaches to a developmental psychopathology model of depression. Development and Psychopathology, 4(1), 189-206.

Harris, A. E., \& Curtin, L. (2002). Parental perceptions, early maladaptive schemas, and depressive symptoms in young adults. Cognitive Therapy and Research, 26(3), 405-416.

Hawke, L. D., Provencher, M. D., \& Arntz, A. (2011). Early maladaptive schemas in the risk for bipolar spectrum disorders. Journal of Affective Disorders, 133(3), 428-436.

Hayes, A. F. (2009). Beyond Baron and Kenny: Statistical mediation analysis in the new millennium. Communication Monographs, 76(4), 408-420.

James, I. A., Reichelt, F. K., Freeston, M. H., \& Barton, S. B. (2007). Schemas as memories: Implications for treatment. Journal of Cognitive Psychotherapy: An International Quarterly, 21(1), 51-57.

Johnson, S. L. (2005). Mania and dysregulation in goal pursuit: A review. Clinical Psychology Review, 25(2), 241-262.

Kaslow, N. J., Deering, C. G., \& Racusin, G. R. (1994). Depressed children and their families. Clinical Psychology Review, 14(1), 39-59.

Keyfitz, L., Lumley, M., Hennig, K., \& Dozois, D. (2013). The role of positive schemas in child psychopathology and resiliency. Cognitive Therapy and Research, 37(1), 97-108.

Kolko, D. J., \& Kazdin, A. E. (1993). Emotional/behavioral problems in clinic and nonclinic children: Correspondence among child, parent and teacher reports. Journal of Child Psychology and Psychiatry and Allied Disciplines, 34(6), 991-1006.

Krahn, G. L., Hohn, M. F., \& Kime, C. (1995). Incorporating qualitative approaches into clinical child psychology research. Journal of Clinical Child Psychology, 24(2), 204-213.

Langer, E. J., \& Roth, J. (1975). Heads I win, tails it's chance: The illusion of control as a function of the sequence of outcomes in a purely chance task. Journal of Personality and Social Psychology, 32(6), 951955.

Larson, R. W. (2000). Toward a psychology of positive youth development. American Psychologist, 55(1), 170-183.

Lobban, F., Taylor, K., Murray, C., \& Jones, S. (2012). Bipolar disorder is a two-edged sword: A qualitative study to understand the positive edge. Journal of Affective Disorders, 141(2-3), 204-212.

Lovejoy, M. C., \& Steuerwald, B. L. (1995). Subsyndromal unipolar and bipolar disorders: Comparisons on positive and negative affect. Journal of Abnormal Psychology, 104(2), 381-384.

Lumley, M. N., Dozois, D. J. A., Hennig, K. H., \& Marsh, A. (2012). Cognitive organization, perceptions of parenting and depression symptoms in early adolescence. Cognitive Therapy and Research, 36(4), 300310.

MacLeod, A. K., \& Byrne, A. (1996). Anxiety, depression, and the anticipation of future positive and negative experiences. Journal of Abnormal Psychology, 105(2), 286-289.

MacLeod, A. K., \& Moore, R. (2000). Positive thinking revisited: Positive cognitions, well-being and mental health. Clinical Psychology and Psychotherapy, 7(1), 1-10.

MacLeod, A. K., \& Salaminiou, E. (2001). Reduced positive future-thinking in depression: Cognitive and affective factors. Cognition and Emotion, 15(1), 99-107.

Masten, A. S. (2007). Resilience in developing systems: Progress and promise as the fourth waive rises. Development and Psychopathology, 19(3), 921-930.

Masten, A. S. (2011). Resilience in children threatened by extreme adversity: Frameworks for research, practice, and translational synergy. Development and Psychopathology, 23(2), 493-506.

Masten, A. S., \& Obradovic, J. (2007). Disaster preparation and recovery: Lessons from research on resilience in human development. Ecology and Society, 13(1), 9.

McClain, L., \& Abramson, L. (1995). Self-schemas, stress, and depressed mood in college students. Cognitive Therapy and Research, 19(4), 419-432.

Michalak, E. E., Yatham, L. N., Kolesar, S., \& Lam, R. W. (2006). Bipolar disorder and quality of life: A patient-centered perspective. Quality of Life Research, 15(1), 25-37.

Neeren, A. M., Alloy, L. B., \& Abramson, L. Y. (2008). History of parenting and bipolar spectrum disorders. Journal of Social and Clinical Psychology, 27(9), 1021-1044.

Nilsson, A. K., Jorgensen, C. R., Straarup, K. N., \& Licht, R. W. (2010). Severity of affective temperament and maladaptive self-schemas differentiate borderline patients, bipolar patients, and controls. Comprehensive Psychology, 51(5), 486-491.

Parker, G. (1983). Parental affectionless control as an antecedent to adult depression: A risk factor delineated. Archives of General Psychiatry, 40(9), 956-960.

Patterson, J. (2002). Understanding family resilience. Journal of Clinical Psychology, 58(3), 223-246. 
Pavuluri, M. N. (2008). What works for Bipolar Kids: Help and Hope for Parents. New York, NY: The Guilford Press.

Pavuluri, M. N., Graczyk, E. A., Henry, D. B., Carbray, J. A., Heidenreich, J., \& Miklowitz, D. (2004). Child and family-focused cognitive behavioral therapy for pediatric bipolar disorder: Development and preliminary results. Journal of the American Academy of Child and Adolescent Psychiatry, 43(5), 528-537.

Pavuluri, M. N., Henry, D. B., Devineni, B., Carbray, J. A., \& Birmaher, B. (2006). Child mania rating scale: Development, reliability, and validity. Journal of the American Academy of Child and Adolescent Psychiatry, 45(5), 550-560.

Preacher, K. J., \& Hayes, A. F. (2004). SPSS and SAS procedures for estimating indirect effects in simple mediation models. Research Methods, Instruments, \& Computers, 36(4), 717-731.

Preacher, K. J., \& Hayes, A. F. (2008). Asymptotic and resampling strategies for assessing and comparing indirect effects in multiple mediator models. Research Methods, 40(3), 879-891.

Prevatt, F. (2003). The contribution of parenting practices in a risk and resiliency model of children's adjustment. The British Journal of Developmental Psychology, 21(4), 469-480.

Prieto, S. L., Cole, D. A., \& Tageson, C. W. (1992). Depressive self-schemas in clinic and nonclinic children. Cognitive Therapy and Research, 16(5), 521-534.

Rosenfarb, I. S., Becker, J., \& Khan, A. (1994). Perceptions of parental and peer attachments by women with mood disorders. Journal of Abnormal Psychology, 103(4), 637-644.

Schenkel, L. S., West, A. E., Harral, E. M., Patel, N. B., \& Pavuluri, M. N. (2008). Parent-child interactions in pediatric bipolar disorder. Journal of Clinical Psychology, 64(4), 422-437.

Schludermann, S., \& Schludermann, E. (1988). Questionnaire for children and youth (CRPBI-30). Winnipeg, MB: University of Manitoba.

Schmidt, N. B., Schmidt, K. L., \& Young, J. E. (1999). Schematic and interpersonal conceptualizations of depression: An integration. In T. Joiner \& J. C. Coyne (Eds.), The interactional nature of depression (pp. 127-148). Washington, DC: American Psychological Association.

Shirk, S., Boergers, J., Eason, A., \& Van Horn, M. (1998). Dysphoric interpersonal schemata and preadolescents' sensitization to negative events. Journal of Clinical Child Psychology, 27(1), 54-68.

Silk, J., Vanderbilt-Adriance, E., Shaw, D. S., Forbes, E. E., Whalen, D. J., et al. (2007). Resilience among children and adolescents at risk for depression: Mediation and moderation across social and neurobiological contexts. Development and Psychopathology, 19(3), 841-865.

Stange, J. P., Hamilton, J. L., Burke, T. A., Kleiman, E. M., O'Garro-Moore, J. K., Seligman, N. D., et al. (2015). Negative cognitive styles synergistically predict suicidal ideation in bipolar spectrum disorders: A 3-year prospective study. Psychiatry Research, 226(1), 162-168.

Taylor, S. E., \& Brown, J. D. (1994). Positive illusions and well-being revisited: Separating fact from fiction. Psychological Bulletin, 116(1), 21-27.

Wagnild, G. M., \& Young, H. M. (1993). Development and psychometric evaluation of the Resilience Scale. Journal of Nursing Measurement, 1(2), 165-178.

Whitman, P. B., \& Leitenberg, H. (1990). Negatively biased recall in children with self-reported symptoms of depression. Journal of Abnormal Child Psychology, 18(1), 15-27.

Wood, A. M., \& Tarrier, N. (2010). Positive clinical psychology: A new version and strategy for integrated research. Clinical Psychology Review, 30(7), 819-129.

Young, J. E., Kolosko, J. S., \& Weishar, M. E. (2003). Schema therapy: A practitioner's guide. New York, NY: Guilford Press.

Youngstrom, E. A., Birmaher, B., \& Findling, R. L. (2008). Pediatric bipolar disorder: Validity, phenomenology, and recommendations for diagnosis. Bipolar Disorder, 10(1), 194-214. 